\title{
A complex intersex condition in a Holstein calf
}

\author{
R. Payan-Carreira ${ }^{\text {a }}$, M.A. Pires ${ }^{\text {a }}$, M. Quaresma ${ }^{\text {b }}$, R. Chaves $^{\mathrm{c}}$, F.

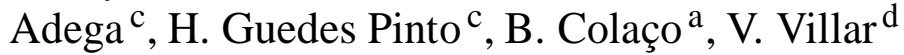 \\ a CECAV, UTAD, Vila Real, Portugal \\ b Veterinary Sciences Department, UTAD, Vila Real, Portugal \\ c Centre of Genetics and Biotechnology, UTAD, Vila Real, Portugal \\ d Department Celular Biology and Anatomy, Facultad de Veterinaria, Leon, Spain
}

\begin{abstract}
A case of disrupted embryonic development of the genital tract in a newborn Holstein calf is described. The physical examination of the calf evidenced several abnormalities, like atresia ani, rudimentary external genitalia and caudal vertebral agenesis. On necropsy, the excised genitalia consisted of bilateral streak gonads, apparently normal uterine tubes, a fluid-filled uterus, a long vagina and a very narrow clitoris-like structure covered with a discrete skin-fold. The urinary tract seemed normal and the urethra's opening was at the vestibule-vaginal junction. A cytogenetic analysis was requested. Karyotype revealed the existence of $\mathrm{Y}$ chromosome material in the two X chromosomes. However, the search for the sex-determining region $\mathrm{Y}$ (SRY) showed that this was an apparently absent gene. The histological examination of the gonads revealed the existence of ovarian dysplasia. Uterine sections evidenced the absence of the uterine epithelium, with only sporadic caruncles. Under microscopic examination, the uterine tubes and vagina structure was normal. The external genitalia sections revealed the existence of a skin-fold covering an erectile structure surrounding the urethra, a structure more similar to a penis than to a clitoris. This is an unusual situation of gonadal dysplasia combined with genital tract anomalies in cattle, probably associated to a genetic defect.
\end{abstract}

Keywords: Congenital anomalies; Ovarian dysplasia; Genital ambiguity; Intersexuality; Cattle

\section{Introduction}

Anomalous development of genitalia has been recognised for long time and frequently described in pigs. These malformations most often concern the female and most anomalies fit in the denomination of intersex (Hunter, 1995; Veitia et al., 2001).

Abnormal development of the reproductive organs occurs with different incidence in several species. Gonadal dysgenesis in a XY-animal results from testicular determination failure and it can be seen in a complete or partial way. In this last situation, some testicular structures develop and generally the individuals exhibit ambiguous genitalia. In the former condition, there is a complete absence of testis formation, and the animals usually develop an integral female phenotype (Vilain et al., 1993; Hunter, 1995; Ahmed and Hughes, 2002). Female XY individuals with gonadal dysgenesis can be related to the absence of the SRY gene or to the inability of SRY transcription (Veitia et al., 2001). In cattle, Freemartin syndrome is the most frequent intersex condition reported (Padula, 2005). A report was recently published concerning a female pseudohermaphrodite heifer with gonadal mosaicism (Takagi et al., 2005).

Generally, gonadal abnormalities in a XY-animal result from failure in testicular determination. The inappropriate differentiation of the embryonic gonad compromises the subsequent development and differentiation stages of the internal and the genital tract organs. 
Atresia ani is a common birth defect in cattle and is often accompanied by other congenital defects of the digestive or the urogenital tracts (Ghanem et al., 2005). In atresia ani, survival appears to mainly depend on early diagnosis, anatomical area affected and successful surgical establishment of a patent intestinal tract.

In the case here reported, authors describe a situation of abnormal genital development in a Holstein newborn calf that evidenced rudimentary and undifferentiated external genitalia, internal female genital ducts and streak gonads. The cytogenetic analysis of this individual demonstrated the existence of Y chromosome material in the X chromosomes, which could explain some of the malformations observed.

\section{Case history and evaluation}

One-day-old Holstein intersex calf, born co-twin with an apparently normal female calf, was presented at the Large Animal Surgery Service at the Veterinary Medical Teaching Hospital of UTAD (Portugal) due to atresia ani. At admission, clinical examination of the calf revealed a complete absence of the tail, agenesis of the anus and rudimentary external genitalia, consisting of a small clitoris-like structure (Fig. 1), covered with a skin-fold. No vulvar labia or prepuce were detected. The external genitalia rudiment was located in a scrotal position, caudal to the mammary glands, and at a considerably lower distance to the area at which the anus should be located, in a normal female. X-ray revealed a partial agenesis of the sacral vertebrae and the caudal vertebral agenesis that led to the suspicion of atresia recti (Fig. 2). A cytogenetic analysis was requested. During surgery to establish the patency of the anus, agenesis of the rectum and hydrometra were detected, and euthanasia was performed.

\section{Cytogenetic analysis}

Chromosome preparations of the calf were made from short-term lymphocyte cultures of whole blood samples using standard protocols (Chaves et al., 2002). After G-banding procedures for chromosome identification, the karyotype of the calf under analysis was organised according to the ISCNDB (2001) recommendations. Very briefly, the results evidenced a karyotype of $2 n=60$, with 29 acrocentric autosome pairs and two sex X chromosomes (medium submetacentrics), which is representative of a female individual.

A Y chromosome-specific probe was prepared by a degenerate oligonucleotide primed PCR (DOP-PCR) from the flow-sorted Y chromosome, using 6MW PCR primers and amplification conditions as previously described (Rabbits et al., 1995).

The other probe was constructed with a DOP-PCR, using genomic DNA of the calf in analysis, and simultaneously another PCR amplification and labelling was made with genomic DNA from a normal male Holstein (for control). After labelling, both probes were blocked with unlabelled genomic DNA from the normal male individual and hybridised in metaphase preparations from the normal male.

In situ hybridisation of the probes to the calf metaphase spreads was performed as previously described (Wienberg et al., 1997). Fluorescent in situ hybridisation results from the normal individual probe revealed no signal on the chromosomes of the normal male, as expected, since its sequences were blocked during procedure; but a prominent painting of the $\mathrm{Y}$ chromosome was observed when using the calf probe, revealing the extra amount of Y material in the calf genome.

\subsection{SRY and amelogenin genes amplification}


PCR specific primers for the SRY and Amelogenin (AMEL) genes were designed, namely SRY A $\left(5^{*}\right.$-TCGTGAACGAAGACGAAAGGGGGC- $\left.3^{*}\right)$, SRY B (5*-GCACAAGAAAGTCCAGGCTCTAAG- $\left.3^{*}\right)$, AMEL A (5*-CAGCCAAACCTCCCTCTGC- $\left.3^{*}\right)$ and AMEL B ( $5^{*}$ CCCGCTTGGTCTTGTCTGTTGC $\left.-3^{*}\right)$, and the reactions were performed according to Phua et al. (2003) and Sullivan et al. (1993), respectively, to search for the presence of these genes in the diseased calf genome and simultaneously from the genomes of a male and female normal Holstein individuals, for control. PCR analysis revealed an apparent absence of the SRY gene in the calf's genome that was also absent in the female control, contrary to the expected PCR band observed in the male control. Amelogenin gene amplification results were opposite to the earlier ones, displaying a clear band observed in the calf and female control and two clear bands in the male control.

\section{Post-mortem examination of the genitalia}

\subsection{Necropsy findings}

Post-mortem gross evaluation of the digestive tract confirmed the partial large bowel agenesis. The urinary tract seemed to have developed normally, the urethra extending from the urinary bladder to the vestibule-vaginal junction. A prominent, fluid-filled uterus was observed (Fig. 3A). The caudal branches of the mesenteric vessels were missing. At necropsy, the female genital tract was removed for evaluation and subsequently fixed in $10 \%$ buffered formalin for histological studies using the routine HE staining.

Gross examination of the entire genitalia showed that the gonads were reduced to a fibrous, ovary-like rudiment (Fig. 3B). Uterine tubes were apparently normal. The uterus and the uterine horns were larger than usual at this age, and distended with a watery fluid (Fig. 3A). The internal folding relief of the uterus and uterine horns was absent, and the internal face of the organ was smooth (Fig. 3C). Detailed evaluation revealed that cervical circular folds were reduced to a very low crest and that the cranial vaginal cavity appeared as being incorporated into the uterine cavity (Fig. 3D). No folding was noticed in the vaginal cavity. The vestibule seemed longer than normal at this age, with the urethral opening near the vestibule-vaginal transition. In the vestibule two portions were found: a smaller cranial portion, with the normal vestibular morphology, and a longer tubular portion, that continued from the former and ended at the narrow external opening. No mucus was present in the vagina or in the vestibular cavity. Analysis of the uterine fluid (density, $\mathrm{pH}$, protein and glucose content and sediment evaluation) showed its compatibility with young calf urine.

External genital structures were very rudimentary and located lower than usual, in a scrotal position. It consisted on a small clitoris-like structure, covered with a skin-fold, lacking vulvar or prepucial appearance.

On a schematic representation (Fig. 4) it is possible to compare the genital malformations found in this animal with the normal genitalia of a calf of the same age, which was also used to compare genital tract measurements (length $\times$ width $\times$ height). In the normal female, ovaries measured $1.5 \mathrm{~cm} \times 0.5 \mathrm{~cm} \times 0.5 \mathrm{~cm}$, the uterine horns $4 \mathrm{~cm} \times 1 \mathrm{~cm}$, the uterine body $4 \mathrm{~cm} \times 0.8 \mathrm{~cm}$, the vagina $7 \mathrm{~cm} \times 4.5 \mathrm{~cm}$ and the vestibule $4 \mathrm{~cm} \times 1.8 \mathrm{~cm}$. In the diseased calf, ovaries measured $1.5 \mathrm{~cm} \times 0.2 \mathrm{~cm} \times 0.1 \mathrm{~cm}$, the uterine horns $17 \mathrm{~cm} \times 8 \mathrm{~cm}$, the uterine body together with the vagina were $5 \mathrm{~cm} \times 10 \mathrm{~cm}$ and the vestibule measured $3 \mathrm{~cm} \times 1.2 \mathrm{~cm}$. The caudal tubular structure measured $10 \mathrm{~cm} \times 0.2 \mathrm{~cm}$. In the normal genitalia, the distance from the upper vagina to the vulvar opening measured $11 \mathrm{~cm}$, while in the abnormal genitalia the distance from the vestibular-vaginal 
transition to the external opening was $13 \mathrm{~cm}$.

\subsection{Histological evaluation of the genital structures}

The histological examination of the fibrous gonadal structure revealed the existence of ovarian dysplasia. Rudimentary gonads presented a poorly defined morphologic boundary between the cortex and inner medulla (Fig. 5A), beside few primordial follicular structures scattered within the cortex that seemed to be degenerated (Fig. 5B). Uterine tubes showed a normal histological structure. On uterine sections, myometrium was normally developed; however, the endometrial glands and the uterine epithelium were absent and only sporadic caruncles could be found near the apex of the uterine horns (Fig. 5C). Cranial vagina and vestibule evidenced normal structure and they bare stratified epithelium.

Histological evaluation of the external genitalia did not confirm the existence of a clitorislike structure, as suspected during gross evaluation of the excised genitalia. Instead, it revealed a phallic configuration (Fig. 5D). The outer layer of this structure was composed of connective tissue, nervous corpuscles and two irregularly organised layers of smooth muscle; the inner layer was composed of fusocellular connective tissue with blood vessels, some of them arranged in cavernous structures, resembling a corpus spongiosum. The urethra was found in the center of this structure. This penis-like structure layed inside a cutaneous pouch, externally covered by haired skin and internally bordered by stratified epithelium.

\section{Discussion}

The case reported here is complex in morphology and rare in occurrence due to the multiple malformations coexisting in the same animal. This article focuses mainly on the genital tract anomalies exhibited by an affected calf, which do not fit either in the Freemartinism syndrome or in the classic XY sex reversal already described (Pailhoux et al., 2001; Padula, 2005). Not even totally fit in other syndrome descriptions commonly associated to external genital dysmorfogenesis as reported for other species (Warot et al., 1997; Mauch and Albertine, 2002). In this case, the classical cytogenetic studies of the calf first revealed a karyotype of $2 n=60, \mathrm{XX}$. The results obtained for the Amelogenin gene were similar to those observed in a female condition. Molecular cytogenetic analysis of this individual revealed the existence of $Y$ chromosome material in the two $\mathrm{X}$ chromosomes. Despite the existence of $\mathrm{Y}$ chromosome material in the two $\mathrm{X}$ chromosomes revealed by the molecular cytogenetic analysis, PCR molecular search for the SRY gene was unsuccessful.

In the male, the presence of the SRY gene in the Y chromosome is required to initiate testicular differentiation pathways and to inhibit female-specific pathways (Capel et al., 1999). When testicular differentiation is not achieved, whether because SRY expression fails to occur or is expressed after the permissive window for induction of the male pathway, gonadal morphogenesis is impaired and germ cells are not prevented to enter meiosis. Consequently, follicular structures are allowed to develop (Albrecht et al., 2000; Veitia et al., 2001), and the embryonic gonad of an XY animal may develop either towards an ovotestis or an ovary. In this animal, and in agreement with the SRY gene absence, no seminiferous tubular structures were observed.

In the absence of SRY gene material, it would be expected that primordial gonad differentiation towards ovary would proceed. Nevertheless, in the case presented herein, a limited differentiation of ovarian structures was noticed accompanying a relative loss of the cortical dominance and a decrease in the number of follicular structures. A similar depletion in germ cells or follicular structures was also reported in certain sex-reversed individuals and in human monosomy X (Vilain 
et al., 1993; Simpson and Rajkovic, 1999; Pailhoux et al., 2001), and it was suggested that a critical threshold of germ cells is needed for adequate ovarian differentiation to occur. When the ovarian pathway is disrupted, as what possibly happened in this case, gonads are reduced to a fibrous streak, located in the position usually assumed by normal ovaries, histologicaly evidencing a decreased germ cells number and a rich fibrous ovarian-like stroma (Simpson and Rajkovic, 1999). As the karyotype of the calf here analysed demonstrated a derived X/Y chromosome, one can hypothesize that the rearrangement could be the reason for a failure in an $\mathrm{X}$-linked differentiation factor.

In this calf, due to the absence of virilization signals and of anti-Müllerian hormone (AMH), Müllerian ducts were allowed to develop. Therefore, normal uterine tubes, a bipartite uterus and a patent cervix were evidenced; also the cranial vagina was normally developed. Diverging from normality, this calf's vestibule seemed to be arranged in two parts: one was similar to the normal female vestibule, hollowed and presenting longitudinal mucosal folds, with the urethral ostium opening near the vaginal transition. The other part presented a tubular arrangement, with a very narrow caliber, running caudally and ending at the rudimentary external penis-like structure. The fluid content of the uterus, or hydrometra, seemed to correspond to the calf's urine that was kept up-stream to this part due to the huge pressure needed to pass this barrier. According to its histological features, this structure corresponds to a rudimentary penis. The development of a phallic structure, caudal to the vestibule, is not easily explainable. In male embryo, morphogenesis of the penis depends on the penile urethral differentiation and elongation. In the female embryo, penile tubular urethra is not formed (Carlson, 2004; Yamada et al., 2006). Without further studies it is not possible to explain what induced, in this case, the proliferation of the urethral groove to form this short tubular structure in an apparently female embryo and in the seemingly absence of virilization signals.

In this calf, the lack of androgens could explain the absence of penis elongation and preputial formation, leading to rudimentary external genitalia in a scrotal position, as it is well known that virilization of the external genitalia begins shortly after testicular differentiation with a rapid increase of the ano-genital distance (Hunter, 1995; Carlson, 2004).

In the individual here described, accompanying the genital abnormalities, digestive tract anomalies (atresia of the rectum and anus) and skeletal malformations (partial agenesis of sacral vertebrae and caudal vertebral agenesis) were also observed. The atresia of the rectum outcomes from an imperfect canalization of the gut and atresia ani is a consequence of the non-perforation of the anal membrane (Carlson, 2004). In 1993, Saperstein sustained that in calves both defects were frequently associated with other malformations, like lack of tail and urogenital defects. This can be explained by the fact that the caudal gut, the tail and the external genitalia are known to share common transcriptional factors that direct its morphogenesis, like the Hox 13 and Sonic hedgehog $(S h h)$ genes. A disruption on these genes expression could explain both the genital and extragenital malformations observed in this animal, as it was demonstrated that such mutations on rodents lead to malformations on those structures, including abnormal external genitalia development, deficient genital duct morphogenesis, severe anomalies in the urinary tract development and rectal abnormalities (Warot et al., 1997; de Santa Barbara and Roberts, 2002).

The case reported here is a fascinating occurrence of a complex series of congenital defects coexisting in a female calf, whose female twin was normal. Although gonadal dysplasia probably results from defects in sex chromosomes, additional studies are needed to completely elucidate the mechanism responsible for the occurrence of all these malformations.

\section{Acknowledgements}


The authors would like to thank Mrs. Mecia Mourão's contribution for the administrative assistance in the manuscript's preparation, Luis Mendanha for the English correction of the manuscript and Mrs. Lígia Bento for the technical assistance. We are also deeply indebted to Professor Joaquin Camón-Urgel for his comments on the manuscript.

\section{References}

Ahmed, S.F., Hughes, I.A., 2002. The genetics of male undermasculinization. Clin. Endocrinol. (Oxf.) 56 (1), 118.

Albrecht, K.H., Capel, B., Washburn, L.L., Eicher, E.M., 2000. Defective mesonephric cell migration is associated with abnormal testis cord development in C57BL/6J XY (Mus domesticus) mice. Dev. Biol. 225 (1), 26-36.

Capel, B., Albrecht, K.H., Washburn, L.L., Eicher, E.M., 1999. Migration of mesonephric cells into the mammalian gonad depends on Sry. Mech. Dev. 84 (1/2), 127-131.

Carlson, B.M., 2004. Urogenital system. In: Human Embryology and Developmental Biology, 3rd Ed. Mosby, Philadelphia, pp. 393-427.

Chaves, R., Adega, F., Santos, S., Heslop-Harrison, J.S., Guedes-Pinto, H., 2002. In situ hybridization and chromosome banding in mammalian species. Cytogenet. Genome Res. 96, 113-116.

de Santa Barbara, P., Roberts, D.J., 2002. Tail gut endoderm and gut/genitourinary/tail development: a new tissue-specific role for Hoxa13. Development 129 (3), 551-561.

Ghanem, M.E., Yoshida, C., Nishibori, M., Nakao, T., Yamashiro, H., 2005. A case of freemartin with atresia recti and ani in Japanese Black calf. Anim. Reprod. Sci. 85 (3/4), 193-199.

Hunter, R.H.F., 1995. Sex Determination Differentiation and Intersexuality in Placental Mammals. Cambridge University Press, Cambridge, pp. 1-310.

ISCNDB, 2001. International System for Chromosome Nomenclature of Domestic Bovids [2000]. In: Di Berardino, D., Di Meo, G.P., Gallagher, D.S., Hayes, H., Iannuzzi, L. (Eds.), Cytogenet. Cell Genet. 92, 283-299.

Mauch, T.J., Albertine, K.H., 2002. Urorectal septum malformation sequence: insights into pathogenesis. Anat. Rec. 268, 405-410.

Padula, A.M., 2005. The freemartin syndrome: an update. Anim. Reprod. Sci. 87 (1/2), 93-109.

Pailhoux, E., Parma, P., Sundstrom, J., Vigier, B., Servel, N., Kuopio, T., Locatelli, A., Pelliniemi, L.J., Cotinot, C., 2001. Time course of female-to-male sex reversal in $38 \mathrm{XX}$ fetal and postnatal pigs. Dev. Dyn. 222 (3), 328 340 .

Phua, A.C.Y., Abdullah, R.B., Mohamed, Z., 2003. A PCR-based sex determination method for possible aplication in caprine gender selection by simultaneous amplification of the Sry and Aml-X genes. J. Reprod. Dev. 49 (4), $307-$ 311.

Rabbits, P., Impey, H., Heppell-Parton, A., Langford, C., Tease, C., Lowe, N., Bailey, D., Ferguson-Smith, M., Carter, N., 1995. Chromosome specific paints from a high resolution flow karyotype of the mouse. Nat. Genet. 9, 369375.

Saperstein, G., 1993. Congenital abnormalities of internal organs and body cavities. Vet. Clin. North Am. (Food Anim. Pract.) 9, 115-125.

Simpson, J.L., Rajkovic, A., 1999. Ovarian differentiation and gonadal failure. Am. J. Med. Genet. 89 (4), $186-200$.

Sullivan, K.M., Mannucci, A., Kimpton, C.P., Gill, P., 1993. A rapid and quantitative DNA sex test: fluorescence-based PCR analysis of XY homologous gene amelogenin. Biotechniques 15, 636-641.

Takagi, M., Yamagishi, N., Oboshi, K., Kageyama, S., Hirayama, H., Minamihashi, A., Sasaki, M., Wijayagunawardane, M.P., 2005. A female pseudohermaphrodite Holstein heifer with gonadal mosaicism. Theriogenology 63 (1), 60 71.

Veitia, R.A., Salas-Cortes, L., Ottolenghi, C., Pailhoux, E., Cotinot, C., Fellous, M., 2001. Testis determination in mammals: more questions than answers. Mol. Cell. Endocrinol. 179 (1/2), 3-16.

Vilain, E., Jaubert, F., Fellous, M., McElreavey, K., 1993. Pathology of 46 XY pure gonadal dysgenesis: absence of testis differentiation associated with mutations in the testis-determining factor. Differentiation 52 (2), 151-159.

Warot, X., Fromental-Ramain, C., Fraulob, V., Chambon, P., Dolle, P., 1997. Gene dosage-dependent effects of the Hoxa13 and Hoxd-13 mutations on morphogenesis of the terminal parts of the digestive and urogenital tracts. Development 124 (23), 4781-4791.

Wienberg, J., Stanyon, R., Nash, W.G., 1997. Conservation of human vs. feline genome organisation revealed by reciprocal 
chromosome painting. Cytogenet. Cell Genet. 77, 211-217.

Yamada, G., Suzuki, K., Haraguchi, R., Miyagawa, S., Satoh, Y., Kamimura, M., Nakagata, N., Kataoka, H., Kuroiwa, A., Chen, Y., 2006. Molecular genetic cascades for external genitalia formation: an emerging organogenesis program. Dev. Dyn. 235 (7), 1738-1752. 


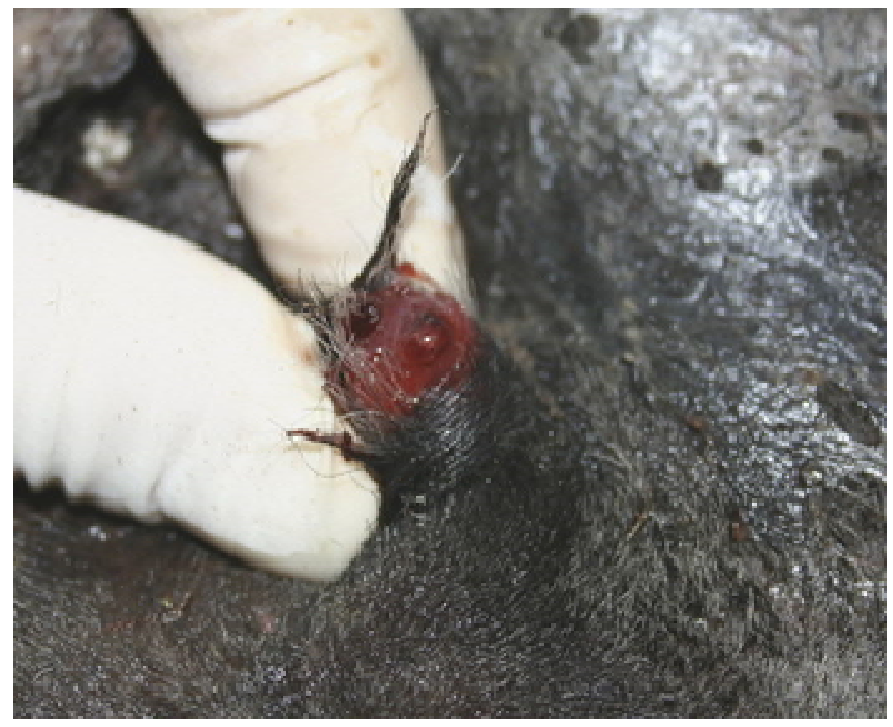

Fig. 1. Rudimentary external genitalia. The external genitalia is reduced to a skin-fold surrounding an erectile structure eccentrically positioned.

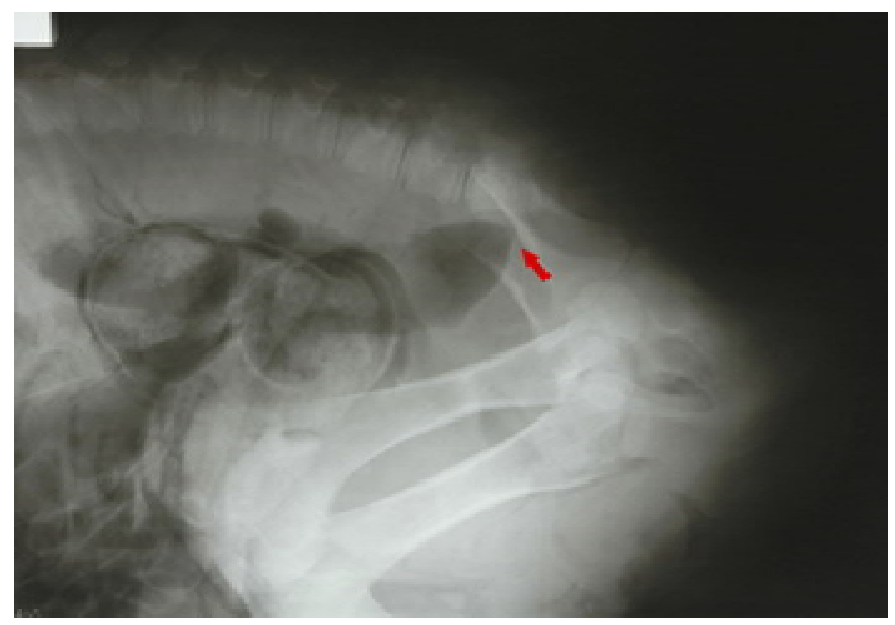

Fig. 2. Radiographic imaging of the caudal abdomen. Agenesis of last sacral and caudal vertebra and atresia recti can be observed. 

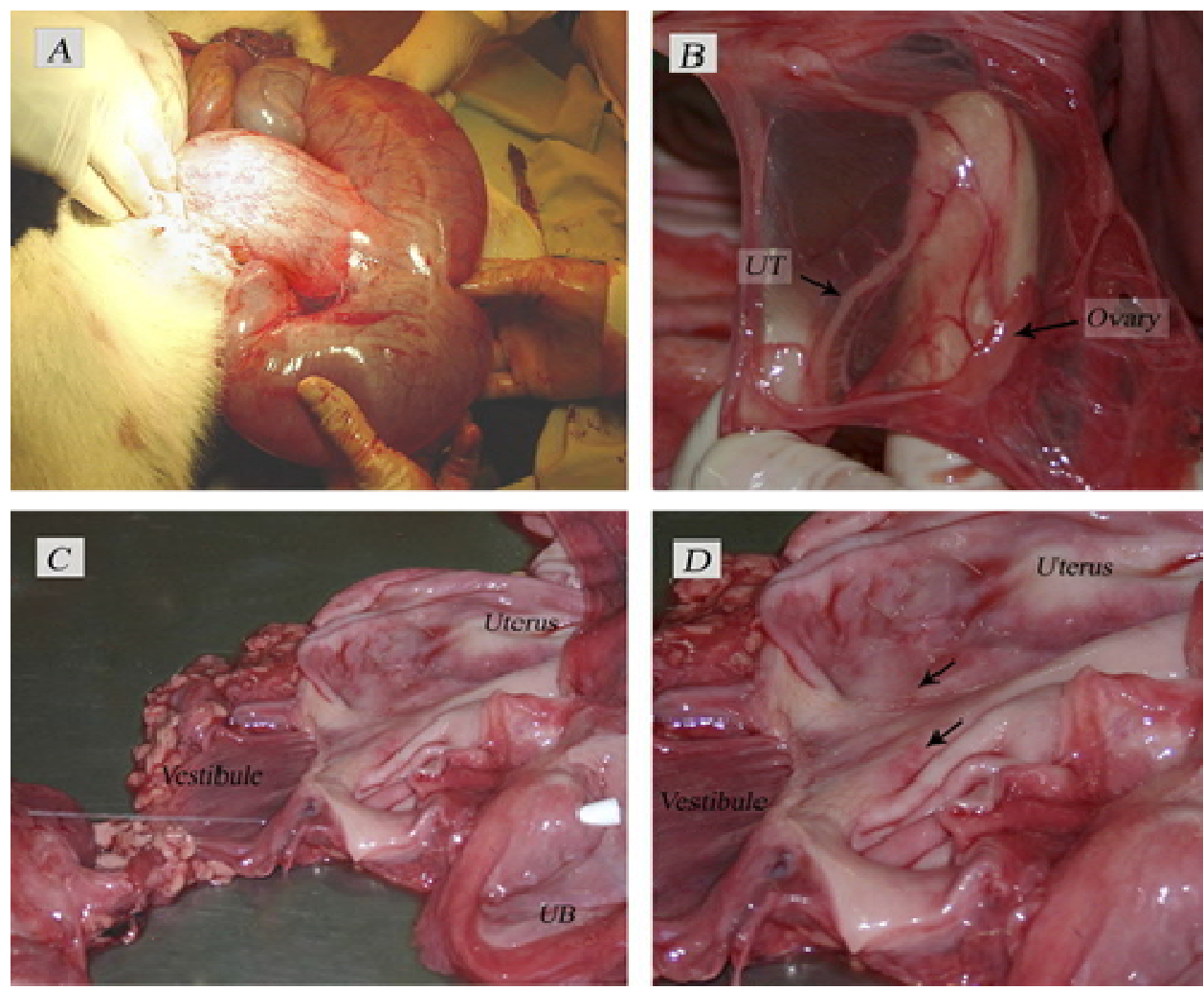

Fig. 3. Macroscopic appearance of the calf genital tract, A; the fluid-filled uterus at the surgery, B; ovary and uterine tube. At gross evaluation, a streak gonad was observed near the uterine tube (UT), C; the uterus, vagina and vestibule evidenced a smooth internal surface. To assess the urethral development, a urinary catheter was inserted through the urethra, from the urinary bladder (UB) to the vestibular-vaginal transition, D; on a closer inspection, cervical remnants (arrows) were detected. 


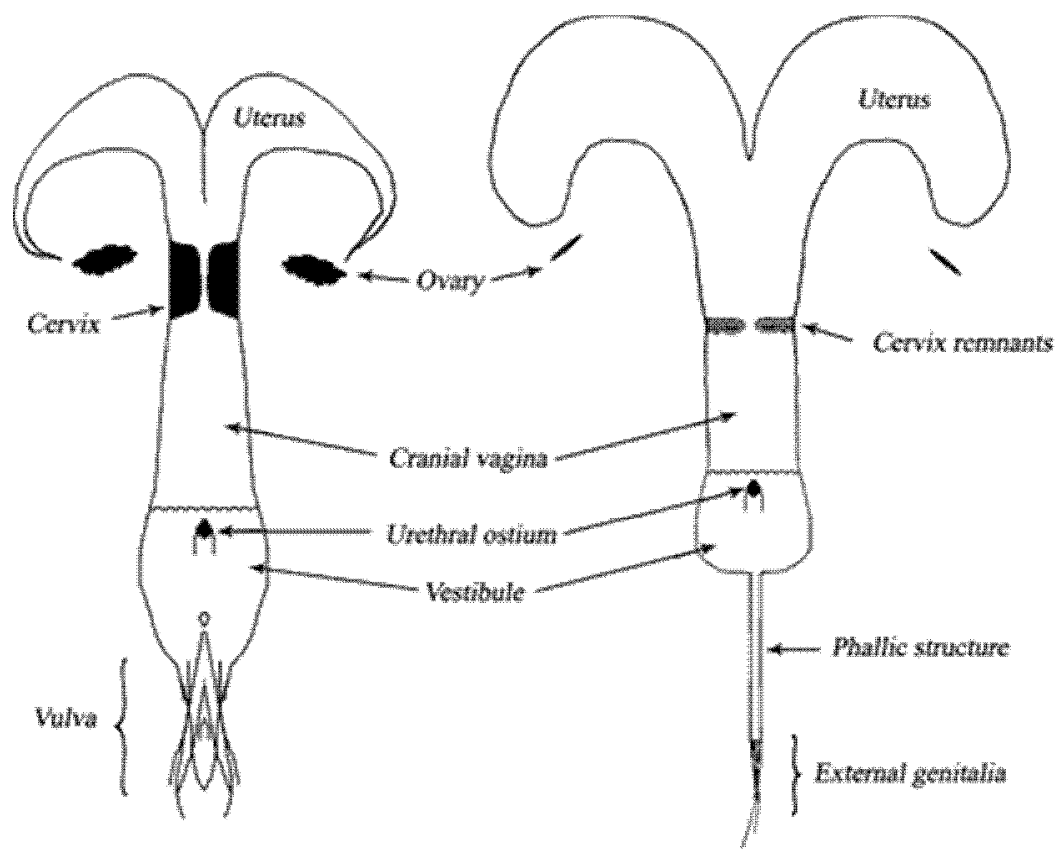

Fig. 4. Schematic representation of both normal bovine genitalia (left) and the excised genitalia from the case reported here (right). The uterine tubes were not depicted in these diagrams. 

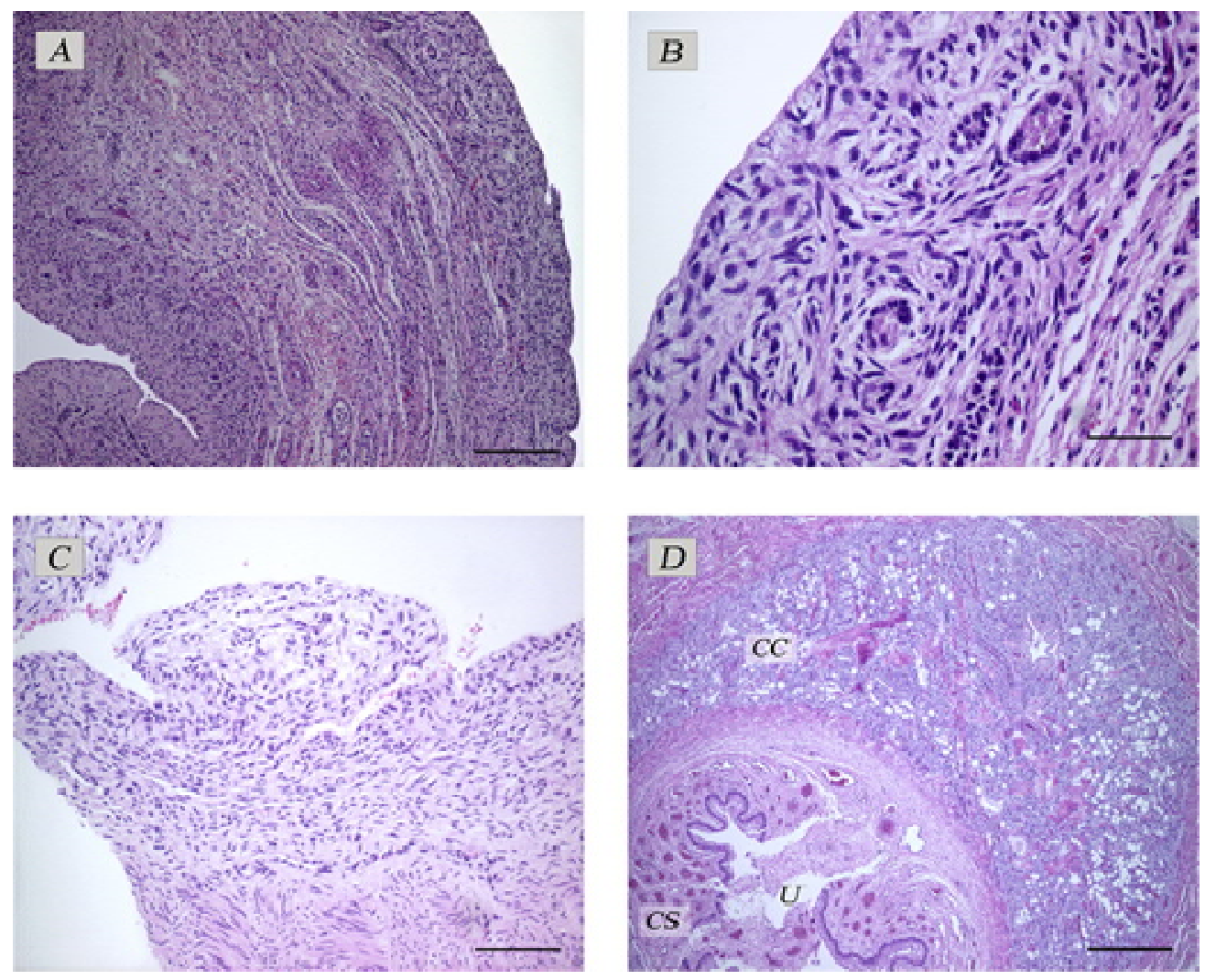

Fig. 5. Histological features, A; ovary (longitudinal section). On lower magnification, the loss of the cortical dominance relatively to the medulla is evident $(\mathrm{HE}$; scale bar $=200 \mathrm{~m}$ [small mu, Greek, m]), B; ovary. On a higher magnification a scarce number of degenerated primordial follicles are observed (HE; scale bar $=50 \mathrm{~m}$ [small mu, Greek, m]), C; uterus. Most of the uterine epithelium is missing from the uterus possibly due to fluid compression of the mucosa. Furthermore, caruncular structures were sporadic in occurrence $(\mathrm{HE}$; scale bar $=100$ $\mathrm{m}$ [small mu, Greek, m]), D; penis. The tubular structure that lies between the vestibule and the external genital rudiment shows a structure compatible with a phallus. The corpus spongiosum (CS) surrounding the urethral mucosa and the corpus cavernosum (CC) covering the dorsolateral aspects of the urethra (U) are evident (HE; scale bar $=500 \mathrm{~m}[$ small mu, Greek, m]). 